\title{
Anne Rolland-Boulestreau, Les notables des Mauges. Communautés rurales et Révolution (1750-1830).
}

\section{Claude Petitfrère}

\section{(2) OpenEdition \\ Journals}

\section{Édition électronique}

URL : https://journals.openedition.org/abpo/1070

DOI : $10.4000 /$ abpo. 1070

ISBN : 978-2-7535-1500-0

ISSN : 2108-6443

\section{Éditeur}

Presses universitaires de Rennes

Édition imprimée

Date de publication : 20 décembre 2005

Pagination : 221-225

ISBN : 978-2-7535-0243-7

ISSN : 0399-0826

\section{Référence électronique}

Claude Petitfrère, «Anne Rolland-Boulestreau, Les notables des Mauges. Communautés rurales et Révolution (1750-1830). », Annales de Bretagne et des Pays de l'Ouest [En ligne], 112-4 | 2005, mis en ligne le 20 décembre 2007, consulté le 22 juillet 2022. URL : http://journals.openedition.org/abpo/1070 ; DOI : https://doi.org/10.4000/abpo.1070 
Rolland-Boulestreau, Anne, Les notables des Mauges. Communautés rurales et Révolution (1750-1830), Rennes, PUR, 2004, 401 p., $22 €$.

Cet ouvrage est tiré d'une thèse élaborée sous la direction de Jean-Clément Martin. Il s'agit d'un travail de micro-histoire, un genre vraisemblablement destiné à se répandre en raison des règlements interdisant aux thésards de multiplier les années d'inscription. Il concerne trois paroisses du nord des Mauges - région que j'ai dénommée naguère le "sanctuaire " de la Vendée angevine Sainte-Christine, Neuvy et Le Pin-en-Mauges, cette dernière illustrée par la figure de Jacques Cathelineau qui fut aux confins du printemps et de l'été 1793, le premier " généralissime " de l'Armée catholique et royale.

Partant, avec raison, de l'idée que la notion d'élite est toujours relative et que chaque communauté en sécrète une à sa mesure, l'auteur a concentré ses recherches sur les notables du premier niveau, ceux qui se dégagent au sein de la paroisse puis de la commune.

Un de ses principaux mérites a été d'envisager cette histoire dans une longue durée englobant les dernières décennies de l'Ancien Régime et les premières décennies du XIXe siècle, ce qui permet de tester à l'échelon local une des thèses partagée par les deux courants historiographiques anciens des insurrections de l'Ouest, antagonistes dans leur parti pris d'exaltation sans nuance de la Vendée pour l'un, de la République pour l'autre, mais qui se rejoignent dans la conception commune d'un immuable caractère propre à la société et à la civilisation locales. De là découle tout naturellement un plan en trois parties chronologiques, l'Ancien Régime, la Révolution, le premier tiers du XIX ${ }^{\mathrm{e}}$ siècle.

Autre mérite de l'auteur, une connaissance satisfaisante de la bibliographie et un souci comparatiste largement démontré, même si parfois citations ou renvois à tel ou tel ouvrage paraissent plaqués artificiellement sur l'exposé.

Anne Rolland-Boulestreau a retenu comme premier indice de la notabilité sous l'Ancien Régime, le témoignage dans les actes de notoriété des archives notariales. Cette démarche soulève plusieurs questions. D'abord la représentativité d'un corpus aussi étroit : 45 témoins figurant dans huit actes de notoriété seulement, échelonnés sur 40 années. Plus de la moitié de ces témoins sont des marchands, mais doit-on en être étonné quand on sait que la plupart des actes semblent en rapport avec le commerce des bestiaux? Plus largement, peuton considérer tous les témoins comme des notables? Ne peut-on imaginer qu'on puisse recourir, pour des raisons de commodité, à des individus d'une moindre notoriété, des voisins du demandeur par exemple? Cependant l'auteur semble confortée dans sa démarche par le fait que les témoins sont gratifiés par le notaire d'avant-noms ou d'épithètes d'honneur. La cause est entendue pour les qualificatifs d'" honnête homme " ou " honorable homme ", ou encore, pour les ecclésiastiques, de "vénérable et discrète personne ", mais on peut être plus circonspect en ce qui concerne "sieur " en position d'avant-nom. L'auteur met d'ailleurs en doute elle-même la portée de tels critères, en soulignant que Jacques Cathelineau, dont la famille est pourtant élevée au rang de " modèle du petit notable ", n'a jamais été gratifié d'aucun avant-nom (p. 95). L'essence de la notabilité, ses composantes en quelque sorte, ressortent bien mieux de quelques études de cas dépourvus d'ambiguïté. Elles montrent que la notoriété est liée au métier (ici on retrouve l'avantage des marchands, concernés plus que d'autres, par la vie de relations), à l'aisance révélée par la place dans la hiérarchie des taillables, et peut-être surtout à des fonctions distinguées au sein de la communauté, celle de fabricien ou de syndic notamment, elles-mêmes en relation avec l'ancienneté de l'implantation familiale dans la paroisse. 
Comptes rendus

À partir de 1789, la quête des notables dans les archives est plus facile dans la mesure où elle repose sur le choix exprimé par la communauté des habitants, ou une partie d'entre elle, lors des élections à l'assemblée de la sénéchaussée, puis des élections municipales du début de l'année 1790. Dans deux cas sur trois les votes confirment purement et simplement la notoriété acquise par les familles sous l'Ancien Régime. Seule Sainte-Christine se distingue en choisissant pour moitié des hommes nouveaux issus de familles implantées assez récemment dans la paroisse. Les élections de novembre 1792 provoquent, quant à elles, un important renouvellement des édiles dans les trois communes - au niveau des officiers municipaux sinon à celui des maires - avec l'entrée en scène de quelques représentants des couches les plus populaires, deux journaliers à Neuvy. Sans doute peut-on voir dans ce phénomène un effet du suffrage universel masculin, mais pour plus de précision, il eût fallu pouvoir disposer des procès-verbaux d'élection malheureusement inexistants. Cependant l'étude minutieuse des liens familiaux permet à Anne Rolland-Boulestreau de montrer que le changement est plus apparent que réel : les nouveaux élus " sont très largement liés à leurs prédécesseurs. Ils se sont fréquentés quotidiennement, ils sont parfois alliés; en tout cas, ils ne semblent pas rivaux " (p. 180). On n'est donc pas étonné que la continuité prédomine dans les choix politiques des municipalités : la mise en œuvre des dispositions de la Constitution civile du clergé, le serment exigé des prêtres, le remplacement des réfractaires, suscitent parmi les nouveaux édiles des trois communes la même hostilité que chez leurs devanciers.

Sur la guerre civile et ses lendemains, l'étude locale confirme le caractère massif d'une révolte qui ne fut pas uniquement paysanne puisque de nombreux artisans ou marchands y participèrent. Caractère massif ne signifie pas unanimité (je n'ai jamais, pour ma part, employé l'expression de "sanctuaire uniforme " [p. 212]). On savait déjà - les cartes publiées dans Les Bleus d'Anjou en témoignent - qu'il se trouva des habitants des Mauges, peu nombreux il est vrai en comparaison de ceux qui s'engagèrent dans le camp opposé, pour choisir la fidélité à la Révolution. Anne Rolland-Boulestreau en donne quelques exemples comme ce Martin Thomas, un tisserand qui se révéla farouche " patriote " après son élection, en 1791, comme juge de paix de Sainte-Christine, ou encore les 13 volontaires du « bataillon des pères de famille " recrutés au début de la guerre civile dans la même commune mais qui y sont, détail à souligner, de nouveaux venus. Elle aurait pu aussi se référer aux travaux concernant les "réfugiés " de la Vendée, qui étaient, pour partie, des "patriotes ", même si la date de sa soutenance ne lui a pas permis de prendre connaissance de la thèse de Guy-Marie Lenne. L'auteur souligne encore, à juste titre, à la fois l'impossible neutralité devant laquelle se trouvaient les habitants des paroisses rurales et l'importance de la pression, physique et psychologique, exercée sur les tièdes ou les timorés par les plus décidés à en découdre : "Il devait être difficile, voire impossible, de refuser de marcher avec les Vendéens, dans un pays où tout le monde se connaît, se côtoie, sous peine d'être mis à l'écart " (p. 225).

À l'époque d'une " reconstruction " qu'Anne Rolland-Boulestreau fait sans doute débuter un peu tôt, en 1795, en négligeant les reprises d'armes ultérieures, celle de 1796 voire 1799, la notion de notabilité aurait pu être bouleversée par le fait que, désormais, elle acquiert une dimension officielle. Même lorsque subsistent des élections, la consécration de l'individu comme notable relève en effet des représentants du pouvoir central, commissaires du Directoire, puis préfets et sous-préfets. Ce contrôle est parfaitement illustré par les atermoiements de l'époque directoriale où l'on voit destituer après le coup d'État anti-royaliste du 18 fructidor an V (4 septembre 1797) d'anciens Vendéens recrutés l'année pré- 
cédente dans les municipalités cantonales. Pourtant, il s'avère que les principaux fondements de la notabilité sous l'Ancien Régime demeurent : c'est une certaine aisance que l'auteur cherche à approcher par les actes d'achats ou de prêt chez les notaires, c'est encore la notoriété acquise par une longue présence de la famille dans la commune et/ou la stabilité d'un établissement dans la même métairie. À lire Anne Rolland-Boulestreau, deux changements considérables seraient pourtant apparus. D'abord l'entrée en lice des familles châtelaines qui figurent au premier plan sur les listes de confiance ou de notabilité et acceptent des places de maires ou font diriger les communes par quelqu'un de leur clientèle. Mais ce changement n'est-il pas illusoire? Les maîtres des châteaux n'ontils pas tout simplement profité de la mise en place d'une structure administrative, les municipalités, qui n'existait pas dans nos campagnes avant la Révolution ou pour être plus précis avant la réforme de 1787? Qui pourrait nier que les nobles, détenteurs de larges pans de la propriété dans les Mauges, plus largement les seigneurs qui, rappelons-le ne sont pas obligatoirement nobles, étaient, avec les curés, les principaux notables ruraux sous l'Ancien Régime? L'autre nouveauté me paraît bien plus réelle. Il s'agit du rôle que le souvenir de la guerre de Vendée a joué dans l'accession à la notabilité au cours du premier XIX siècle. L'auteur a eu la bonne idée de reprendre les dossiers de pension adressés à la monarchie restaurée par d'anciens Vendéens ou leur famille. Elle montre que l'élaboration de ces dossiers fut une occasion pour les communautés de se réapproprier leur passé, en faisant une place centrale aux anciens officiers vendéens ou simplement aux anciens combattants qui, s'étant particulièrement illustrés pour la défense de la "Cause ", étaient devenus ipso facto des notables (p. 300). Cela ressort de l'étude des témoins aux mariages, qui appartiennent en priorité, à d'anciennes familles vendéennes (cependant pouvait-il en être autrement dans des communes si majoritairement " blanches "?), et surtout du fait, nettement plus probant, que les héros locaux de la Vendée sont, plus souvent que d'autres, sollicités comme témoins dans les mariages, des cérémonies qui ont sans doute contribué, comme d'ailleurs d'autres fêtes ou rassemblements populaires, à entretenir la flamme du souvenir vendéen.

La micro-histoire porte en elle un risque bien connu, dès qu'on prétend tirer du particulier un loi générale. Peut-on, à bon droit fonder une règle sur des échantillons minuscules? Dans le cas qui nous occupe, les trois paroisses parlent-elles pour l'ensemble des Mauges comme veut le faire penser le titre? En fait, des travaux plus généraux, nombreux déjà, nous avaient donné les lignes de force de l'histoire vendéenne, et il se trouve que le présent ouvrage non seulement ne les contredit pas mais les conforte. Il ne faut donc pas y chercher de " révélations " bouleversantes. En revanche l'approche micro-historique permet d'illustrer de façon précise des connaissances déjà acquises. Elle autorise également des avancées sur des aspects de l'histoire jusque là peu ou mal explorées. Grâce à la relecture minutieuse de sources connues, comme les interrogatoires de prisonniers par le juge de paix de Chalonnes ou les commissions militaires, les listes des 27 premiers compagnons de Cathelineau établies au XIX ${ }^{\mathrm{e}}$ siècle, grâce au dépouillement d'archives peu souvent utilisées comme celles du diocèse, de certaines paroisses, ou encore celles du château du Lavouer auxquelles Anne Rolland-Boulestreau a eu la chance d'avoir accès, elle a pu jeter un éclairage nouveau sur certains épisodes de la révolte ou de ses antécédents immédiats, comme les rassemblements secrets de La Poitevinière en avril-mai 1792 ou les premières prises d'armes au Pin-en-Mauges. Elle met à mal le mythe d'un Cathelineau providentiellement sorti de l'anonymat en mars 1793 pour mener les gens du Pin au combat, en rappelant que, situation rare dans ce pays 
sous-alphabétisé, Jacques avait bénéficié d'une certaine instruction lors des cinq années passées à la cure de La Chapelle-du-Genet, montrant surtout que, de longue date, sa famille avait acquis notoriété et influence au sein de la paroisse par l'exercice des charges de fabricien et de " sacriste ». Par le dépouillement systématique des registres paroissiaux puis de ceux de l'état civil elle a reconstitué patiemment la parentèle de ses petits notables, et établi les réseaux d'amicalité jaugés à l'aune de la présence aux mariages. Ainsi démontre-t-elle, par exemple, le fort enracinement des premiers compagnons de Cathelineau dans la paroisse et l'existence de liens familiaux pour une majorité d'entre eux, élément essentiel de la cohésion du groupe. Il resterait pourtant à faire la contreépreuve : n'obtiendrait-on pas les mêmes résultats s'agissant des individus qui ne faisaient pas partie de cette première bande d'insurgés? Mais à mon sens le meilleur apport du travail d'Anne Rolland-Boulestreau - on n'en sera pas étonné s'agissant d'une disciple de Jean-Clément Martin - est la mise à jour du seul élément vraiment nouveau dans l'établissement des notoriétés locales au XIX ${ }^{\mathrm{e}}$ siècle, un passé, glorieux autant que possible, de Vendéen.

L'ouvrage est donc loin d'être dépourvu d'intérêt. Pour autant, il n'est pas possible de passer sous silence certaines erreurs ou confusions vraiment regrettables. Ainsi on ne saurait employer l'un pour l'autre " rural " et " paysan " (p. 44 et 46), ni faire des métayers (qui sont ici, rappelons-le, les locataires à redevance fixe - en fait des fermiers - des exploitations les plus importantes) des " propriétaires-exploitants " alors même que l'on donne la durée de leur passage dans la métairie (p. 254). Des églises paroissiales comme celles de Neuvy, Saint-Lézin, Sainte-Christine, La Poitevinière ne peuvent être qualifiées de "succursales " (p. 136). Il ne faut pas confondre la " réserve " et la " directe " des seigneuries (p. 283, 286), ni faire des nobles de tous les seigneurs. Il serait étonnant à ce propos que les Gontard qui appartiennent à une famille d'avocats et d'officiers seigneuriaux avant d'acquérir eux-mêmes un petit fief aient jamais été anoblis sous l'Ancien Régime et dans le cas contraire il eût fallu le dire (282-283). N'est-il pas abusif de ranger, dans une même catégorie de "gens de la terre " ou " agriculteurs " les grands propriétaires rentiers du sol, nobles ou non, et les métayers (p. 250)? Les réalités politiques sont quelquefois malmenées elles aussi. On ne peut parler d'engagement "républicain " pour les municipalités élues en 1790 (p. 219), Bonaparte n'était pas encore devenu Napoléon en 1801 (p. 234), le Conseil des Cinq-Cents n'existe plus sous l'Empire (p. 278). Lorsque Symphorien Brunet est qualifié d'" ex-notable " en 1796-1797 dans des documents concernant les personnes susceptibles de remplir des places dans les municipalités cantonales (p. 47-48), cela fait certainement référence à une fonction officielle antérieure (rappelons que la loi du 14 décembre 1789 instituait dans les municipalités des " notables " à côté des officiers municipaux) et non à une simple position honorable dans l'échelle sociale sous l'Ancien Régime.

On regrettera aussi les trop nombreuses maladresses d'écriture. Citons, entre autres, " les parties géographiques angevines " (p. 94), les métayers " doublés " par les artisans et les marchands (p. 115), des "familles ayant joué des fonctions " (132) ou encore tel individu qui se trouve "à l'écart des sources " (p. 221). Plus grave, les formules grammaticalement fautives ou dépourvues de sens : « le principal est fructifié " (p. 34); un notable "irradiant quatre paroisses " (p. 40); "la structure socioprofessionnelle des communautés coïncide peut-être avec l'élection des premières équipes municipales " (100); « la parentèle [...] détient sans doute encore une place, face aux scrutins de 1790 " (p. 100); " fiscalité et pouvoirs politiques semblent synonymes semble-t-il » (p. 112); " [...] la fabrique apparaît bien synonyme de la détention d'un pouvoir [...] " (p. 123); " cet éche- 
lon désintéresse l'État " (p. 245). Une idée mal exprimée peut induire un contresens : dire que Charles Tilly aurait évoqué les rivalités au sein des communautés " dans une présentation contre-révolutionnaire " (p. 95) fait de lui, bien à tort, un auteur partisan, engagé dans la célébration de la Contre-Révolution. Les titres ou sous-titres eux-mêmes ne sont pas exempts de formules sibyllines voire fausses : "La parentèle, attribut du petit notable "; " Frontières villageoises et guerre de Vendée : les délimitations du territoire "... Il est certain qu'une écriture moins hâtive, une relecture attentive du texte par son auteur ou une autre personne compétente auraient pu éviter l'accumulation de telles scories qui nuisent à la facilité et à l'agrément de la lecture et risquent même de décourager le lecteur.

Claude PETITFRERE

Luc, Jean-Noël (dir.), Histoire de la Maréchaussée et de la Gendarmerie - Guide de recherche, Préface du général d'armée Guy Parayre, Directeur général de la Gendarmerie nationale, Paris, Service historique de la Gendarmerie nationale, 2005, 1108 p.

Les guides de recherche scientifiques sont des instruments dont on ne louera jamais assez l'utilité - les regrets se portant seulement en direction des domaines où ils n'existent pas. On connaît les services rendus à l'histoire de la justice par Jean-Claude Farcy à travers Deux siècles d'histoire de la justice ou Guide des archives judiciaires et pénitentiaires, ou dans un autre domaine par le Guide de l'histoire locale d'Alain Croix et Didier Guyvarc'h.

L'Histoire de la maréchaussée et de la gendarmerie - Guide de recherche, publié en 2005 sous la direction de Jean-Noël Luc et édité par le Service historique de la Gendarmerie nationale est de ce point de vue un événement pour au moins trois raisons : la matière explorée, l'exhaustivité du guide, la clarté de la présentation.

On sait à quel point l'histoire de la gendarmerie a été longtemps négligée par l'histoire scientifique, comme d'ailleurs celle de la police, alors que l'histoire " militaire " a une longue tradition derrière elle - même si on ne la fait plus tout à fait aujourd'hui comme hier. On sait aussi combien le développement des recherches sur la gendarmerie - et la maréchaussée - a été fulgurant ces dernières années grâce notamment à l'étroite collaboration du SHGN et de l'équipe du Centre d'histoire du XIX ${ }^{\mathrm{e}}$ siècle, principalement animé ici par Jean-Noël Luc (Paris IV). De cette explosion résulte entre autres cet énorme volume, fruit d'un travail de plusieurs années et dont on ne peut que féliciter avec une admiration parfois un peu étonnée les multiples auteurs, sous-officiers, officiers de gendarmerie et universitaires (ils peuvent participer des deux qualités).

Le titre Guide de recherche pèche d'ailleurs peut-être un peu, mais à vrai dire s'il pèche, c'est par sa modestie. Le volume dépasse de loin en effet le statut de simple guide bibliographique ou archivistique, ce qui aurait été déjà fort utile. En effet, après une brève mais précise introduction de J.-N. Luc faisant le point sur cet immense chantier, l'ouvrage se divise en six parties, - L'institution, Bibliographie, Archives, Autres sources, Lieux de mémoire, Dictionnaire historique. À la fois donc un guide, un dictionnaire, autre genre d'outil en plein développement, et une sorte d'almanach aux multiples informations pratiques : comment désormais se passer de la chronologie de l'histoire de l'institution qui occupe les pages 115 à 188 ? 